\title{
First observations on Prefab posets' Whitney numbers
}

\section{A. Krzysztof Kwaśniewski}

the Dissident - relegated by Białystok University authorities from the Institute of Computer Science to Faculty of Physics ul. Lipowa 41, 15424 Białystok, Poland e-mail: kwandr@gmail.com

Summary We introduce a natural partial order $\leq$ in structurally natural finite subsets of the cobweb prefabs sets recently constructed by the present author. Whitney numbers of the second kind of the corresponding subposet which constitute Stirling-like numbers' triangular array - are then calculated and the explicit formula for them is provided. Next - in the second construction - we endow the set sums of prefabiants with such an another partial order that their their Bell-like numbers include Fibonacci triad sequences introduced recently by the present author in order to extend famous relation between binomial Newton coefficients and Fibonacci numbers onto the infinity of their relatives among which there are also the Fibonacci triad sequences and binomial-like coefficients (incidence coefficients included). The first partial order is $F$ sequence independent while the second partial order is $F$-sequence dependent where $F$ is the so called admissible sequence determining cobweb poset by construction. An $F$-determined cobweb poset's Hasse diagram becomes Fibonacci tree sheathed with specific cobweb if the sequence $F$ is chosen to be just the Fibonacci sequence.

AMS Classification Numbers: 05C20, 11C08, 17B56 . Key Words: prefab, exponential structure, cobweb poset, Whitney numbers, Bell-like numbers, Fibonacci-like sequences

presented (November 2006) at the Gian-Carlo Rota Polish Seminar

http://ii.uwb.edu.pl/akk/sem/sem_rota.htm

published: Advances in Applied Clifford Algebras

Volume 18, Number 1 / February, 2008, 57-73

ONLINE FIRST, Springer Link Date, Friday, August 10, 2007

\section{Introduction}

The clue algebraic concept of combinatorics - the so called prefab (with associative and commutative composition) was introduced in [1], see also [2,3]. The elements of prefabs are called since now on - prefabiants. In [4] the present author had constructed a new broader class of prefab's extending combinatorial structure based on the so called cobweb posets (see Section 1. [4] for the definition of a cobweb poset as well as a combinatorial interpretation of its characteristic binomial-type coefficients for example- fibonomial ones $[5,6])$.

Here we introduce two natural partial orders: one $\leq$ in grading-natural subsets of cobweb's prefabs sets [4] and in the second proposal we endow the set sums of prefabiants with such another partial order that one may extend the Bell numbers to sequences of Bell-like numbers encompasing among infinity of others the Fibonacci triad sequences introduced by the present author in [7]. 


\section{Prefab based posets and their Whitney num- bers.}

Let the family $S$ of combinatorial objects (prefabiants) consists of all layers $\left\langle\Phi_{k} \rightarrow\right.$ $\left.\Phi_{n}\right\rangle, \quad k<n, \quad k, n \in N=0,1,2, \ldots$ and an empty prefabiant $i$.

The set $\wp$ of prime objects consists of all sub-posets $\left\langle\Phi_{0} \rightarrow \Phi_{m}\right\rangle$ i.e. all $P_{m}$ 's $m \in N$ constitute from now on a family of prime prefabiants which we define after[4] in two steps. Namely accompanying the set $E$ of edges to the set $V$ of vertices - one obtains the Hasse diagram where here down $p, q, s \in N$. (Convention: Edges stay for arrows directed - say - upwards - see examples below).

Definition 1

$P=\langle V, E\rangle, \quad V=\bigcup_{0 \leq p} \Phi_{p}, \quad E=\{\langle\langle j, p\rangle,\langle q,(p+1)\rangle\rangle\} \bigcup\{\langle\langle 1,0\rangle,\langle 1,1\rangle\rangle\}, \quad$ where $1 \leq j \leq p_{F}, 1 \leq q \leq(p+1)_{F}$

. The finite cobweb sub-poset $P_{m}$ is then defined accodingly.

Definition $2 P_{m}=\left\langle V_{m}, E_{m}\right\rangle$, where $V_{m}=\bigcup_{0 \leq s \leq m} \Phi_{s}$ and $E_{m}$ is defined as $E$ restricted to $V_{m}$ by $1 \leq p \leq m-1$ is called the prime cobweb poset.

Layer

$$
\left\langle\Phi_{k} \rightarrow \Phi_{n}\right\rangle
$$

is considered here to be the set of all max-disjoint isomorphic copies (iso-copies) of $P_{m}, m=n-k[4]$. As a matter of illustration we quote after [4] examples of cobweb posets' Hasse Diagrams [9] so that the layers become visualized.

\section{and so on up}

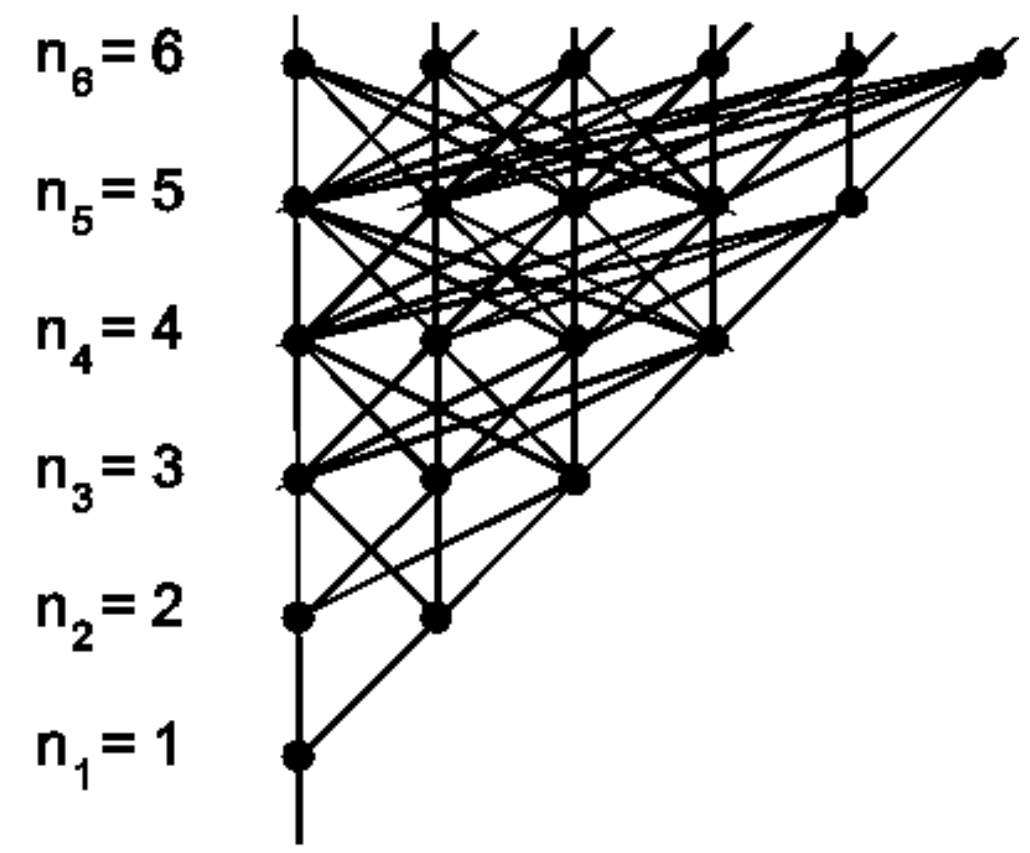


Fig.1. Display of Natural numbers' cobweb poset.

and so on up

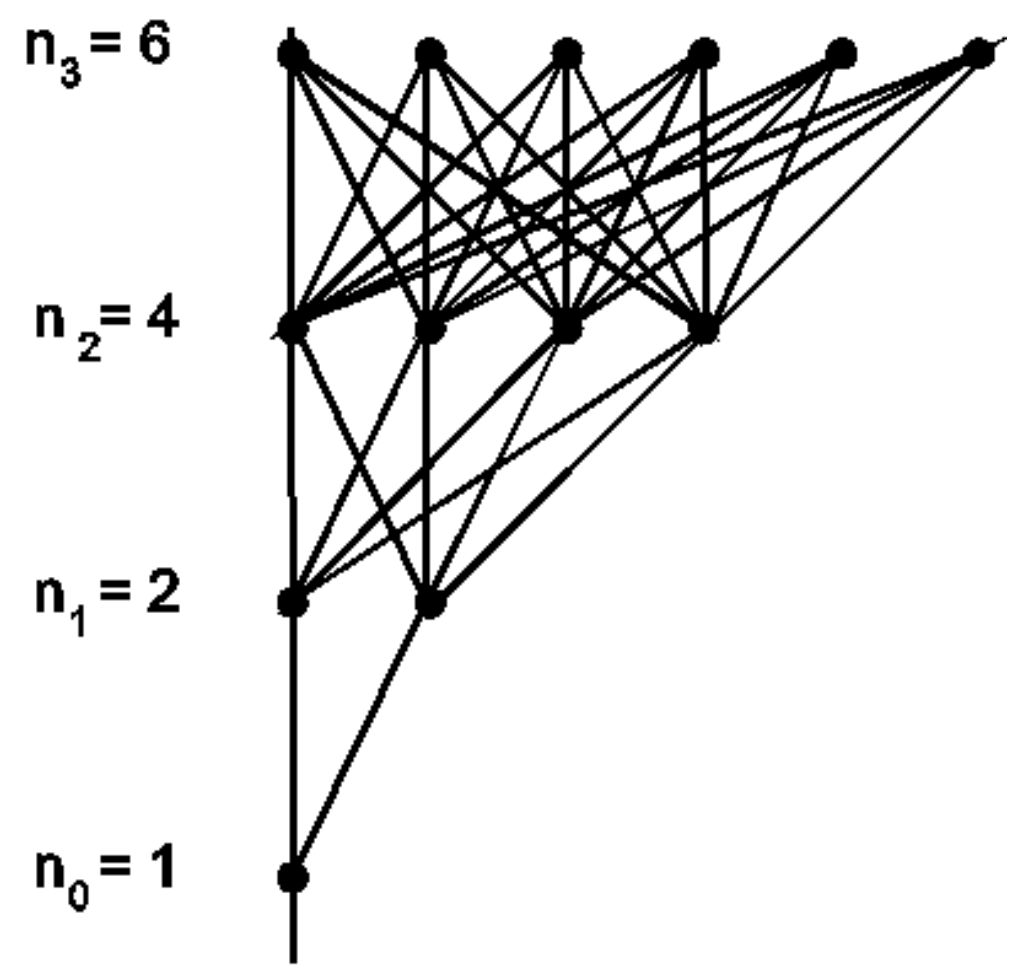

Fig.2. Display of Even Natural numbers $\cup\{1\}$-cobweb poset. 


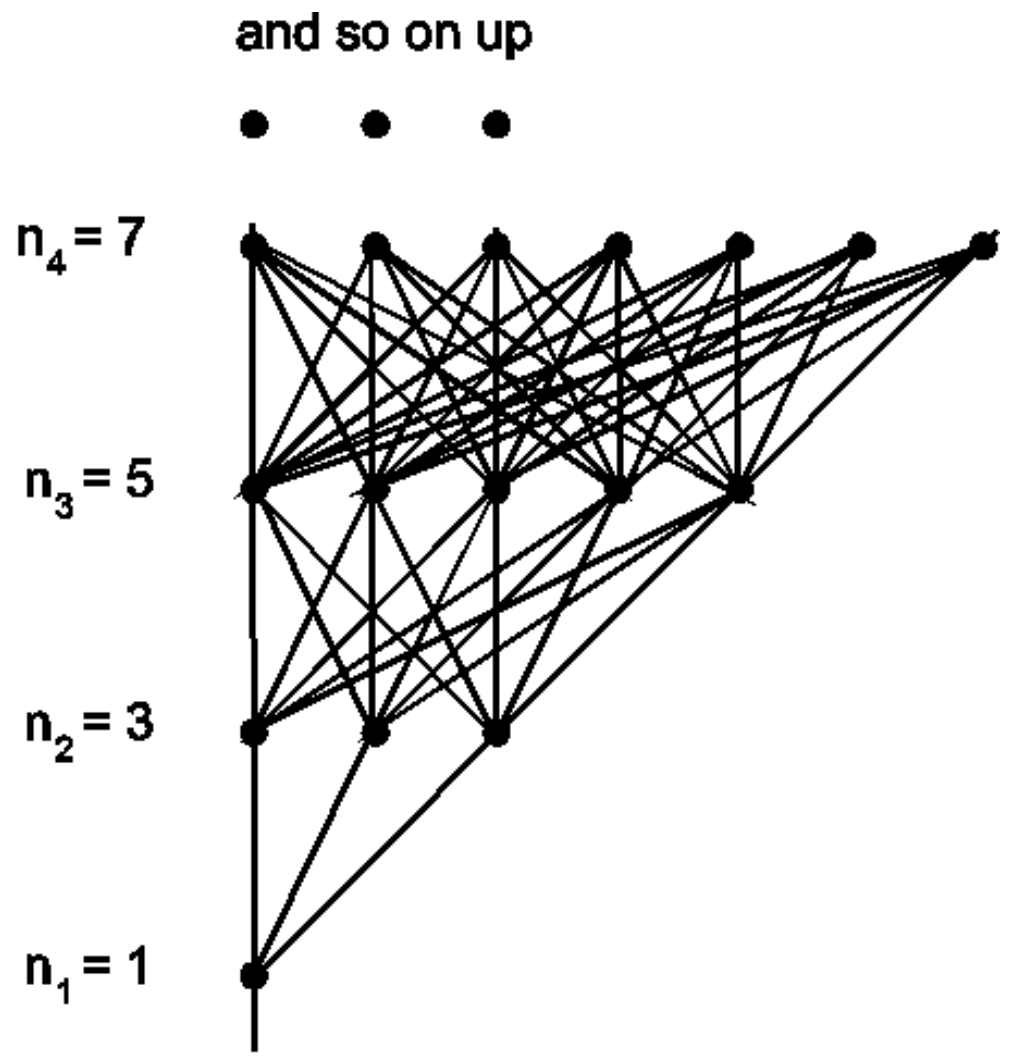

Fig3. Display of Odd natural numbers' cobweb poset. and so on up

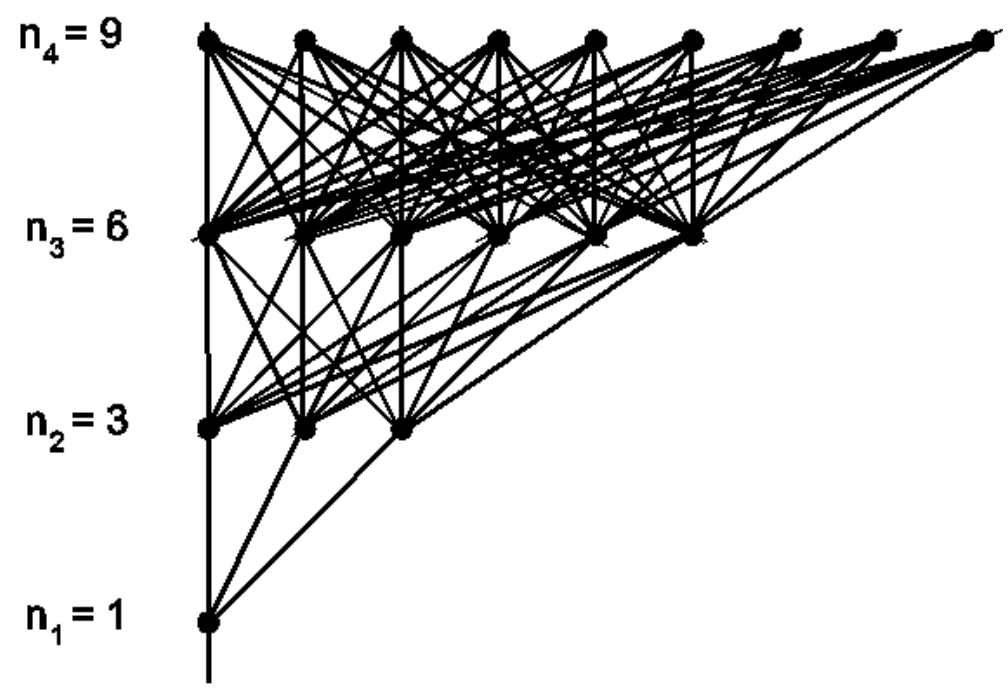

Fig.4. Display of divisible by 3 natural numbers $\cup\{1\}$ - cobweb poset. 


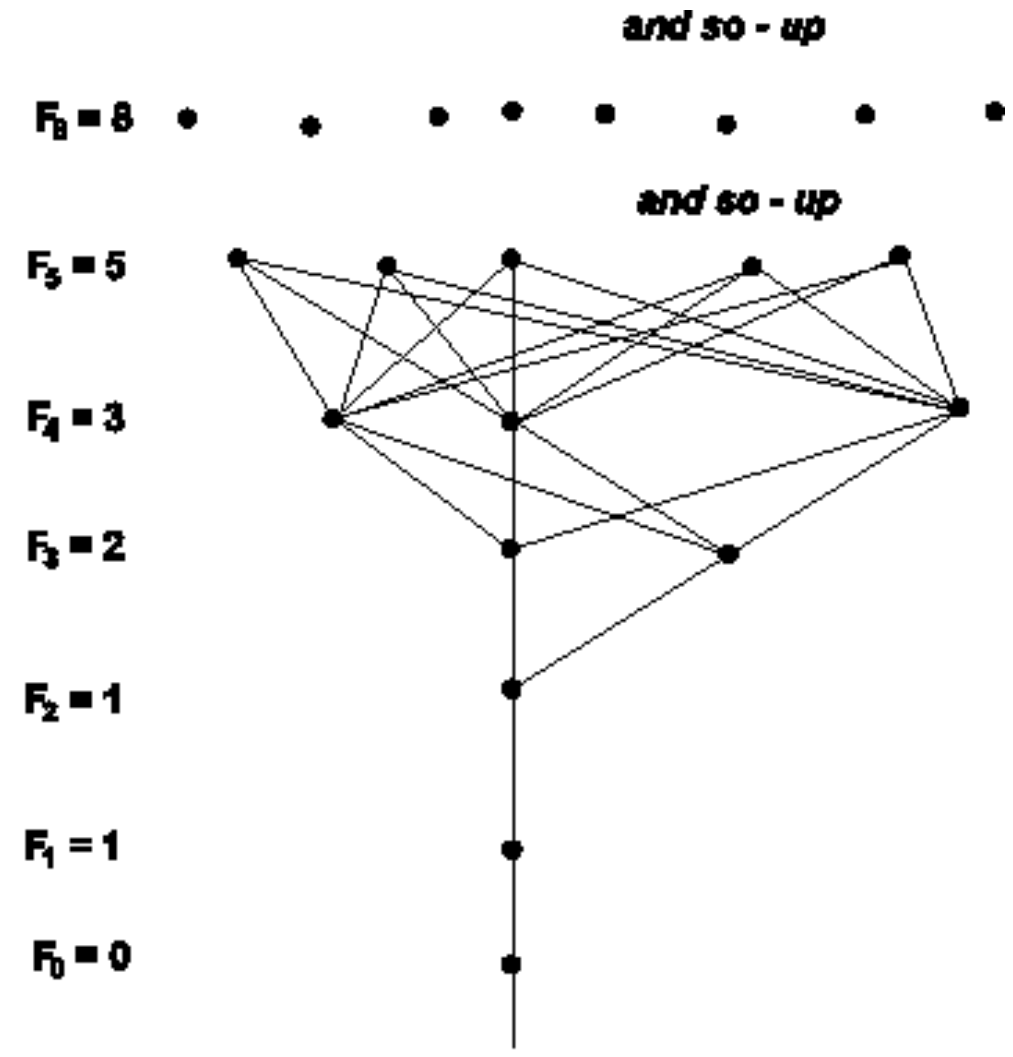

Fig.5. Display of Fibonacci numbers' cobweb poset.

\section{Cobweb posets ${ }^{6}$ combinatorial interpretation}

As seen above - for example the Fig.5. displays the rule of the construction of the Fibonacci "cobweb" poset. It is being visualized clearly while defining this [non-lattice!] cobweb poset $P$ with help of its incidence matrix [8]. The incidence $\zeta$ function matrix representing uniquely just this cobweb poset $P$ has the staircase structure correspondent with "cobwebed" Fibonacci Tree i.e. a Hasse diagram [9] of the particular partial order relation under consideration. 


$$
\left[\begin{array}{lllllllllllllllll}
1 & 1 & 1 & 1 & 1 & 1 & 1 & 1 & 1 & 1 & 1 & 1 & 1 & 1 & 1 & 1 & \ldots \\
0 & 1 & 1 & 1 & 1 & 1 & 1 & 1 & 1 & 1 & 1 & 1 & 1 & 1 & 1 & 1 & \ldots \\
0 & 0 & 1 & 1 & 1 & 1 & 1 & 1 & 1 & 1 & 1 & 1 & 1 & 1 & 1 & 1 & \ldots \\
0 & 0 & 0 & 1 & 0 & 1 & 1 & 1 & 1 & 1 & 1 & 1 & 1 & 1 & 1 & 1 & \ldots \\
0 & 0 & 0 & 0 & 1 & 1 & 1 & 1 & 1 & 1 & 1 & 1 & 1 & 1 & 1 & 1 & \ldots \\
0 & 0 & 0 & 0 & 0 & 1 & 0 & 0 & 1 & 1 & 1 & 1 & 1 & 1 & 1 & 1 & \ldots \\
0 & 0 & 0 & 0 & 0 & 0 & 1 & 0 & 1 & 1 & 1 & 1 & 1 & 1 & 1 & 1 & \ldots \\
0 & 0 & 0 & 0 & 0 & 0 & 0 & 1 & 1 & 1 & 1 & 1 & 1 & 1 & 1 & 1 & \ldots \\
0 & 0 & 0 & 0 & 0 & 0 & 0 & 0 & 1 & 0 & 0 & 0 & 0 & 1 & 1 & 1 & \ldots \\
0 & 0 & 0 & 0 & 0 & 0 & 0 & 0 & 0 & 1 & 0 & 0 & 0 & 1 & 1 & 1 & \ldots \\
0 & 0 & 0 & 0 & 0 & 0 & 0 & 0 & 0 & 0 & 1 & 0 & 0 & 0 & 1 & 1 & \ldots \\
0 & 0 & 0 & 0 & 0 & 0 & 0 & 0 & 0 & 0 & 0 & 1 & 0 & 1 & 1 & 1 & \ldots \\
0 & 0 & 0 & 0 & 0 & 0 & 0 & 0 & 0 & 0 & 0 & 0 & 1 & 1 & 1 & 1 & \ldots \\
0 & 0 & 0 & 0 & 0 & 0 & 0 & 0 & 0 & 0 & 0 & 0 & 0 & 1 & 0 & 0 & \ldots \\
0 & 0 & 0 & 0 & 0 & 0 & 0 & 0 & 0 & 0 & 0 & 0 & 0 & 0 & 1 & 0 & \ldots \\
0 & 0 & 0 & 0 & 0 & 0 & 0 & 0 & 0 & 0 & 0 & 0 & 0 & 0 & 0 & 1 & \ldots \\
. & . & . & . & . & . & . & . & . & . & . & . & . & . & . & . & \ldots
\end{array}\right]
$$

Figure 6. The incidence matrix $\zeta$ for the Fibonacci cobweb poset

Note The knowledge of $\zeta$ matrix explicit form enables one to construct (count) via standard algorithms [8] the Möbius matrix $\mu=\zeta^{-1}$ and other typical elements of incidence algebra perfectly suitable for calculating number of chains, of maximal chains etc. in finite sub-posets of $P$. All elements of the corresponding incidence algebra are then given by a matrix of the Fig.6 with 1's replaced by any reals ( or ring elements in more general cases).

We have a natural combinatorial object characterizig the cobweb posets Hasse directed graphs.

Namely - in general ([4-7], [10], [12], [13]) - given any sequence $\left\{F_{n}\right\}_{n \geq 0}$ of nonzero reals one may define its corresponding binomial-like $F$ - nomial coefficients in the spirit of Ward's Calculus of sequences [13]s as follows

\section{Definition 3}

$$
\left(\begin{array}{c}
n \\
k
\end{array}\right)_{F}=\frac{F_{n} !}{F_{k} ! F_{n-k} !} \equiv \frac{n \frac{k}{F}}{k_{F} !}, \quad n_{F} \equiv F_{n} \neq 0, n \geq 0
$$

where we make an analogy driven identifications in the spirit of Ward's Calculus of sequences $\left(0_{F} \equiv 0\right)$ :

$$
\begin{aligned}
& n_{F} ! \equiv n_{F}(n-1)_{F}(n-2)_{F}(n-3)_{F} \ldots 2_{F} 1_{F} ; \\
& 0_{F} !=1 ; \quad n \frac{k}{F}=n_{F}(n-1)_{F} \ldots(n-k+1)_{F} .
\end{aligned}
$$

This is just the adaptation of the notation for the purpose Fibonomial Calculus case (see references in [4-7], [10], 12]).

The crucial and elementary observation now is that the cobweb poset combinatorial interpretation of $F$-binomial coefficients $[4-7,10,12,14,16]$ makes sense not for arbitrary $F$ sequences as $F-$ nomial coefficients should be nonnegative integers.

Definition 4 A sequence $F=\left\{n_{F}\right\}_{n \geq 0}$ is called cobweb-admissible iff

$$
\left(\begin{array}{c}
n \\
k
\end{array}\right)_{F} \in N \quad \text { for } \quad k, n \in N
$$


Right from the definition of $P$ via its Hasse diagram here now follow quite obvious and important observations. They lead us to a combinatorial interpretation of cobweb poset's characteristic binomial-like coefficients (for example - fibonomial ones $[6,16]$ ). Here they are with the first obvious observation at the start.

\section{4, Observation 1}

The number of maximal chains starting from The Root (level $0_{F}$ ) to reach any point at the $n-$ th level with $n_{F}$ vertices is equal to $n_{F}$ !.

Observation $2(k>0)$

The number of all maximal chains in-between $(k+1)-$ th level $\Phi_{k+1}$ and the $n-t h$ level $\Phi_{n}$ with $n_{F}$ vertices is equal to $n_{F}^{\frac{m}{F}}$, where $m+k=n$.

Indeed. Denote the number of ways to get along maximal chains from any fixed point (the leftist for example) in $\Phi_{k}$ to $\Rightarrow \Phi_{n}, n>k$ with the symbol

$$
\left[\Phi_{k} \rightarrow \Phi_{n}\right]
$$

then obviously we have :

$$
\left[\Phi_{0} \rightarrow \Phi_{n}\right]=n_{F} !
$$

and

$$
\left[\Phi_{0} \rightarrow \Phi_{k}\right] \times\left[\Phi_{k} \rightarrow \Phi_{n}\right]=\left[\Phi_{0} \rightarrow \Phi_{n}\right]
$$

In order to formulate the combinatorial interpretation of $F$ - sequence - nomial coefficients (F-nomial - in short) let us consider all finite "max-disjoint" sub-posets rooted at the $k-t h$ level at any fixed vertex $\langle r, k\rangle, 1 \leq r \leq k_{F}$ and ending at corresponding number of vertices at the $n$-th level $(n=k+m)$ where the "max-disjoint" sub-posets are defined below.

Definition 5 Two families of maximal chains including two equipotent copies of $P_{m}$ are said to be max-disjoint if considered as sets of maximal chains they are disjoint i.e they have no maximal chain in common. (All $P_{m}$ 's constitute from now on a family of the so called prime [4,10] prefabiants). An equipotent copy of $P_{m}$ ['equip-copy'] is defined as such a family of maximal chains equinumerous with $P_{m}$ set of maximal chains that the it constitues a sub-poset with one minimal element.

Definition 6 We denote the number of all max-disjoint equipotent copies of $P_{m}$ rooted at any vertex $\langle j, k\rangle, 1 \leq j \leq k_{F}$ of $k-$ th level with the symbol

$$
\left(\begin{array}{l}
n \\
k
\end{array}\right)_{F} \text {. }
$$

One uses the customary convention: $\left(\begin{array}{l}0 \\ 0\end{array}\right)_{F}=1$.

Naturally- let us recall- the above definition makes sense not for arbitrary $F$ sequences as $F-$ nomial coefficients should be nonnegative integers i.e. the sequence $F=\left\{n_{F}\right\}_{n \geq 0}$ must be cobweb-admissible.

Problem 0. The partition or tiling problem. Suppose now that $F$ is a cobweb admissible sequence. Let us introduce

$$
\sigma P_{m}=C_{m}\left[F ; \sigma<F_{1}, F_{2}, \ldots, F_{m}>\right]
$$


the equipotent sub-poset obtained from $P_{m}$ with help of a permutation $\sigma$ of the sequence $<F_{1}, F_{2}, \ldots, F_{m}>$.

Then

$$
P_{m}=C_{m}\left[F ;<F_{1}, F_{2}, \ldots, F_{m}>\right] .
$$

Consider the layer $\left\langle\Phi_{k} \rightarrow \Phi_{n}\right\rangle, \quad k<n, \quad k, n \in N$. Layer is considered here to be the set of all max-disjoint equipotent copies of $P_{n-k}$. The question then arises, whether and under which conditions the layer may be partitioned with help of maxdisjoint blocks of the form $\sigma P_{m}$. At first - this main question answer is in affirmitave. Some computer experiments done by student Maciej Dziemiańczuk [17] are encouraging However problems : "how many?" or "find it all" are still opened.

Recall now that the number of ways to reach an upper level from a lower one along any of maximal chains i.e. the number of all maximal chains from the level $\Phi_{k+1}$ to $\Rightarrow \Phi_{n}, \quad n>k$ is equal to

$$
\left[\Phi_{k} \rightarrow \Phi_{n}\right]=n_{F}^{\frac{m}{F}}
$$

Naturally then we have

$$
\left(\begin{array}{c}
n \\
k
\end{array}\right)_{F} \times\left[\Phi_{0} \rightarrow \Phi_{m}\right]=\left[\Phi_{k} \rightarrow \Phi_{n}\right]=n \frac{m}{F}
$$

where $\left[\Phi_{0} \rightarrow \Phi_{m}\right]=m_{F}$ ! counts the number of maximal chains in any equip-copy of $P_{m}$. With this in mind we see that the following holds.

\section{Observation $3(\mathbf{n}, \mathbf{k} \geq \mathbf{0})$}

Let $n=k+m$. Let $F$ be any cobweb admissible sequence. Then the number of max-disjoint equip-copies i.e. sub-posets equipotent to $P_{m}$, rooted at the same fixed vertex of $k-$ th level and ending at the $n$-th level is equal to

$$
\begin{aligned}
& \frac{n \frac{m}{F}}{m_{F} !}=\left(\begin{array}{c}
n \\
m
\end{array}\right)_{F} \\
= & \left(\begin{array}{c}
n \\
k
\end{array}\right)_{F}=\frac{n \frac{k}{F}}{k_{F} !} .
\end{aligned}
$$

Note The above Observation 3 provides us with the new combinatorial interpretation of the class of all classical $F$ - nomial coefficients including distinguished binomial or distinguished Gauss $q$ - binomial ones or Konvalina generalized binomial coefficients of the first and of the second kind [11,12]- which include Stirling numbers too. The vast family of Ward-like [13] admissible by $\psi=\left\langle\frac{1}{n_{F} !}\right\rangle_{n \geq 0}$-extensions $F$-sequences $[12,14,16]$ includes also those desired here which shall be called "GCDmorphic" sequences. This means that $G C D\left[F_{n}, F_{m}\right]=F_{G C D[n, m]}$ where $G C D$ stays for Greatest Common Divisor operator. The Fibonacci sequence is a much ontrivial $[16,6]$ and guiding famous example of GCD-morphic sequence. Naturally incidence coefficients of any reduced incidence algebra of full binomial type [8] are GCD-morphic sequences therefore they are now independently given a new cobweb combinatorial interpretation via Observation 3. More on that - see the next section where prefab combinatorial description is being served. Before that - on the way - let us formulate the following problem (opened?).

Problem 1 Find effective characterizations of the cobweb admissible sequence i.e. find all examples.

Note on admissibility. Observation 3 from [16] provides us with the new combinatorial interpretation of the class of all classical $F$ - nomial coefficients including 
distinguished binomial or distinguished Gauss $q$ - binomial ones or Konvalina generalized binomial coefficients of the first and of the second kind [11]- which include Stirling numbers too. This vast family of Ward-like [13] cobweb admissible $F$-sequences - admissible at first by the so called $\psi=\left\langle\frac{1}{n_{F} !}\right\rangle_{n \geq 0}$ umbral extensions [9] - includes also those desired here which shall be called $" G C D$-morphic" sequences.

Definition 7 The sequence of integers $F=\left\{n_{F}\right\}_{n \geq 0}$ is called the GCD-morphic sequence if $G C D\left[F_{n}, F_{m}\right]=F_{G C D[n, m]}$ where $G C D$ stays for Greatest Common Divisor operator.

Recall again : the Fibonacci sequence is a much nontrivial [6] and guiding example of GCD-morphic sequence . Naturally incidence coefficients of any reduced incidence algebra of full binomial type [8] are cobweb-admissible. Question: which of these above are GCD-morphic sequences?

In view of the Note on admissibility the following problems are apparently interesting also on their own.

Characterization Problem Find effective characterizations of the cobweb admissible sequence i.e. find all examples.

GCD-morphism Problem Find effective characterizations i.e. find all examples.

\section{Prefabs 6 Whitney numbers}

Consider then now the partially ordered family $S$ of these layers considered to be sets of all max-disjoint isomorphic copies (iso-copies) of prime prefabiants $P_{m}=P_{n-k}$ as displayed by Fig 1. - Fig.5. examples above. For any $F$-sequence determining cobweb poset let us define in $S$ the same partial order relation as follows.

\section{Definition 8}

$$
\left\langle\Phi_{k} \rightarrow \Phi_{n}\right\rangle \leq\left\langle\Phi_{k *} \rightarrow \Phi_{n}\right\rangle \equiv k \leq k^{*} \wedge n \leq n^{*} .
$$

For convenience reasons we shall also adopt and use the following notation:

$$
\left\langle\Phi_{k} \rightarrow \Phi_{n}\right\rangle=p_{k, n}
$$

The interval $\left[p_{k, n}, p_{k} *, n\right]$ is of course a subposet of $\langle S, \leq\rangle$. We shall consider in what follows the subposet $\left\langle P_{k, n}, \leq\right\rangle$ where

$$
P_{k, n}=\left[p_{o, o}, p_{k, n}\right] .
$$

Observation 1. The size $\left|P_{k, n}\right|$ of $P_{k, n}=\mid\{\langle l, m\rangle, \quad 0 \leq l \leq k \wedge \quad 0 \leq m \leq$ $n \wedge k \leq n\} \mid=(n-k)(k+1)+\frac{k(k+1)}{2}$.

Proof: Obvious. Just draw the picture $\{\langle l, m\rangle, \quad 0 \leq l \leq k \wedge 0 \leq m \leq n \wedge k \leq$ $n$ \} of $P_{k, n}$ ' grid.

Observation 2. The number of maximal chains in $\left\langle P_{k, n}, \leq\right\rangle$ is equal to the number $d(k, n)$ of 0 - dominated strings of binary i.e. $0^{\prime} s$ and $1^{\prime} s$ sequences

$$
d(k, n)=\frac{n+1-k}{n}\left(\begin{array}{c}
k+n \\
n
\end{array}\right) .
$$

Proof. The number we are looking for equals to the number of minimal walk-paths in $[k \times n]$ Manhattan grid [15] - paths restricted by the condition $k \leq n$ i.e. it equals to the number of 0 - dominated strings of $0^{\prime} s$ and $1^{\prime} s$ sequences. 
Recall that $(d(k, n))$ infinite matrix's diagonal elements are equal to the Catalan numbers $C(n)$

$$
C(n)=\frac{1}{n}\left(\begin{array}{c}
2 n \\
n
\end{array}\right) .
$$

as the Catalan numbers count the number of 0 - dominated strings of $0^{\prime} s$ and $1^{\prime} s$ with equal number of $0^{\prime} s$ and $1^{\prime} s$. Recall that a 0 - dominated string of length $n$ is such a string that the first $k$ digits of the string contain at least as many $0^{\prime} s$ as $1^{\prime} s$ for $k=1, \ldots, n$ i.e. 0 's prevail in appearance, dominate $1^{\prime} s$ from the left to the right end of the string. 0 - dominated strings correspond bijectively to minimal bottom - left corner to the right upper corner paths in an integer grid $Z_{\geq} \times Z_{\geq}$rectangle part called

Manhattan [15] with the restriction imposed on those minimal paths to obey the "safety" condition $k \leq n$.

Comment 1. Observation 2. equips the poset $\left\langle P_{k, n}, \leq\right\rangle$ with clear cut combinatorial meaning.

The poset $\left\langle P_{k, n}, \leq\right\rangle$ is naturally graded. $\left\langle P_{k, n}, \leq\right\rangle$ poset's maximal chains are all of equal size (Dedekind property) therefore the rang function is defined.

Observation 3. The rang $r\left(P_{k, n}\right)$ of $P_{k, n}=$ number of elements in maximal chains $P_{k, n}$ minus one $=k+n-1$. The rang $r\left(p_{l, m}\right)$ of $\pi=p_{l, m} \in P_{k, n}$ is defined accordingly: $r\left(p_{l, m}\right)=l+m-1$.

Proof: obvious. Just draw the picture $\{\langle l, m\rangle, \quad 0 \leq l \leq k \quad \wedge \quad 0 \leq m \leq n \quad \wedge \quad k \leq$ $n\}$ of $P_{k, n}$ ' grid and note that maximal means paths without at a slant edges.

Accordingly Whitney numbers $W_{k}\left(P_{l, m}\right)$ of the second kind are defined as follows (association: $n \leftrightarrow\langle l, m\rangle$ )

\section{Definition 9}

$$
W_{k}\left(P_{l, m}\right)=\sum_{\pi \in P_{l, m}, r(\pi)=k} 1 \equiv S(k,\langle l, m\rangle) .
$$

Here now and afterwords we identify $W_{k}\left(P_{l, m}\right)$ with $S(k,\langle l, m\rangle)$ called and viewed at as Stirling - like numbers of the second kind of the naturally graded poset $\left\langle P_{k, n}, \leq\right\rangle$ - note the association: $n \leftrightarrow\langle l, m\rangle$.

\section{Right now challenge problems. I.}

I. Let us define now Whitney numbers $w_{k}\left(P_{l, m}\right)$ of the first kind as follows (association: $n \leftrightarrow\langle l, m\rangle$. Note the text-book notation for Möbius function $\mu$ )

\section{Definition 10}

$$
w_{k}\left(P_{l, m}\right)=\sum_{\pi \in P_{l, m}, r(\pi)=k} \mu(0, \pi) \equiv s(k,\langle l, m\rangle) .
$$

Here now and afterwards we identify $w_{k}\left(P_{l, m}\right)$ with $s(k,\langle l, m\rangle)$ called and viewed at as Stirling - like numbers of the first kind of the poset $\left\langle P_{k, n}, \leq\right\rangle$ - note the association: $n \leftrightarrow\langle l, m\rangle$.

Problem 1 Find an explicit expression for

$$
w_{k}\left(P_{l, m}\right) \equiv s(k,\langle l, m\rangle)=?
$$

and

$$
W_{k}\left(P_{l, m}\right) \equiv S(k,\langle l, m\rangle)=?
$$


Occasionally note that $S(k,\langle l, m\rangle)$ equals to the number of the grid points counted at a slant (from the up-left to the right-down) accordingly to the $l+m=k$ requirement.

Problem 2 Find the recurrence relations for

$$
w_{k}\left(P_{l, m}\right) \equiv s\left(k,\langle l, m\rangle \quad \text { and } \quad W_{k}\left(P_{l, m}\right) \equiv S(k,\langle l, m\rangle) .\right.
$$

We define now (note the association: $n \leftrightarrow\langle l, m\rangle$ ) the corresponding Bell-like numbers

$$
B(\langle l, m\rangle)
$$

of the naturally graded poset $\left\langle P_{k, n}, \leq\right\rangle$ as follows.

\section{Definition 11}

$$
B(\langle l, m\rangle)=\sum_{k=0}^{l+m} S(k,\langle l, m\rangle) .
$$

\section{Observation 4.}

$$
B(\langle l, m\rangle)=\left|P_{l, m}\right|=\frac{k(k+1)}{2}+(n-k)(k+1) .
$$

Proof: Just draw the picture $\{\langle l, m\rangle, \quad 0 \leq l \leq k \wedge 0 \leq m \leq n \wedge k \leq n\}$ of $P_{k, n}$ ' grid and note that $S(k,\langle l, m\rangle)$ equals to the number of the grid points counted at a slant (from the up-left to the right-down) accordingly to the $l+m=k$ requirement. Summing them up over all gives the size of $P_{k, n}$.

Comment 2. Observation 4. equips the poset's $\left\langle P_{k, n}, \leq\right\rangle$ Bell-like numbers $B(\langle l, m\rangle)$ with clear cut combinatorial meaning.

\section{Set Sums of prefabiants ${ }^{6}$ posets and their Whit- ney numbers.}

In this part we consider prefabiants' set sums with an appropriate another partial order so as to arrive at Bell-like numbers including Fibonacci triad sequences introduced recently by the present author in [16] - see also $[7,6]$.

Let $F$ be any "GCD-morphic" sequence. This means that $G C D\left[F_{n}, F_{m}\right]=F_{G C D[n, m]}$ where $G C D$ stays for Greatest Common Divisor mapping. We define the $F$-dependent finite partial ordered set $P(n, F)$ as the set of prime prefabiants $P_{l}$ given by the sum below.

\section{Definition 12}

$$
P(n, F)=\bigcup_{0 \leq p}\left\langle\Phi_{p} \rightarrow \Phi_{n-p}\right\rangle=\bigcup_{0 \leq l} P_{n-l}
$$

with the partial order relation defined for $n-2 l \leq 0$ according to

\section{Definition 13}

$$
P_{l} \leq P_{\hat{l}} \equiv l \leq \hat{l}, \quad P_{\hat{l}}, P_{l} \in\left\langle\Phi_{l} \rightarrow \Phi_{n-l}\right\rangle .
$$

Recall that rang of $P_{l}$ is $l$. Note that $\left\langle\Phi_{l} \rightarrow \Phi_{n-l}\right\rangle=\emptyset$ for $n-2 l \leq 0$. The Whitney numbers of the second kind are introduce accordingly.

\section{Definition 14}

$$
W_{k}\left(P_{n, F}\right)=\sum_{\pi \in P_{n, F}, r(\pi)=k} \equiv S(n, k, F) .
$$


Right from the definitions above we infer that: (recall that rang of $P_{l}$ is $l$.)

Observation 5.

$$
W_{k}\left(P_{n, F}\right)=\sum_{\pi \in P_{n, F}, r(\pi)=k} \equiv S(k, n-k, F)=\left(\begin{array}{c}
n-k \\
k
\end{array}\right)_{F} .
$$

Here now and afterwords we identify $W_{k}\left(P_{n, F}\right)=S(n, k, F)$ viewed at and called as Stirling - like numbers of the second kind of the $P$ defined in [10]. $P$ by construction (see Figures above) displays self-similarity property with respect to its prime prefabiants sub- posets $P_{n}=P(n, F)$.

Right now challenge problems. II.

We repeat with obvious replacements of corresponding symbols, names and definitions the same problems as in "Right now challenge problems. I".

Here now consequently - for any $G C D$-morphic sequence $F$ (see: [10]) we define the corresponding Bell-like numbers $B_{n}(F)$ of the poset $P(n, F)$ as follows.

\section{Definition 15}

$$
B_{n}(F)=\sum_{k \geq 0} S(n, k, F) .
$$

Due to the investigation in $[7,16]$ we have right now at our disposal all corresponding results of $[16,7]$ as the following identification with special case of $\langle\alpha, \beta, \gamma\rangle$ - Fibonacci sequence $\left\langle F_{n}^{[\alpha, \beta, \gamma]}\right\rangle_{n \geq 0}$ defined in [7] holds.

Observation 6 .

$$
B_{n}(F) \equiv F_{n+1}^{[\alpha=0, \beta=0, \gamma=0]}
$$

Proof: See the Definition 2.2. from [7]. Compare also with the special case of formula (6) in [16].

Recurrence relations. Recurrence relations for $\langle\alpha, \beta, \gamma\rangle$ - Fibonacci sequences $F_{n}^{[\alpha, \beta=, \gamma]}$ are to be found in [7] - formula (9). Compare also with the special case formula (7) in [16].

Closing-Opening Remark. The study of further properties of these Bell-like numbers as well as the study of consequences of these identifications for the domain of the widespread data types [7] and perhaps for eventual new dynamical data types we leave for the possibly coming future. Examples of special cases - a bunch of them - one finds in [7] containing [16] as a special case. As seen from the identification Observation 6. the special cases of $\langle\alpha, \beta, \gamma\rangle$ - Fibonacci sequences $F_{n}^{[\alpha, \beta, \gamma]}$ gain additional with respect to $[16,7]$ combinatorial interpretation in terms Bell-like numbers as sums over rang $=k$ parts of the poset i.e. just sums of Whitney numbers of the poset $P(n, F)$. This adjective "additional" shines brightly over Newton binomial connection constants between bases $\left\langle(x-1)^{k}\right\rangle_{k \geq 0}$ and $\left\langle x^{n}\right\rangle_{n \geq 0}$ as these are Whitney numbers of the numbers from $[n]$ chain i.e. Whitney numbers of the poset $\langle[n], \leq\rangle$. For other elementary "shining brightly" examples see Joni, Rota and Sagan excellent presentation in [18].

\section{On applications of new cobweb posets ${ }^{6}$ origi- $^{-}$ nated Whitney numbers}

Applications of new cobweb posets' originated Whitney numbers such as extended Stirling or Bell numbers are expected to be of at least such a significance in applications to linear algebra of formal series [linear algebra of generating functions [19]] as Stirling and Bell numbers or their $q$-extended correspondent already are in the so called coherent physics [20] ( see [20] also for abundant references on the subject). Also 
straightforward applications of prefabs to coherent physics [20] are on line. [Quantum coherent states physics is of course a linear theory with its principle of states' superposition].

In order to say more on the subject of this section and give some examples let us remind the equivalence of exponential structures by Stanley [21] with corresponding exponential prefabs [1]

In this context the let us indicate the crucial "Ward'ian - prefab'ian" example we owe to Gessel [22] with his $q$-analog of the exponential formula as expressed by the Theorem 5.2 from [22].

We also recall that the $q$-analog of the Stirling numbers of the second kind investigated by Morrison in Section 3 of [23] constitute the same example of Ward'ian prefab'ian extension as in the Bender - Goldman - Wagner Ward - prefab example. As noticed there by Morrison the $(\gamma-e . g . f$. $)$ prefab exponential formula may equally well be derived from the corresponding Stanley's exponential formula in [21]. Let us then now come over to these exponential structures of Stanley with an expected impact on the current considerations ( for definitions, theorems etc. see [21]). In this connection we recall quoting (notation from [21]) an important class of Stanley's Stirling - like numbers $\frac{S_{n k}}{M(n)}$ of the second and those of the first kind Stanley's Stirling - like numbers $\frac{s_{n k}}{M(n)}$. Both kinds are characteristic immanent for counting of exponential structures (or equivalently - corresponding exponential prefabs) and inheriting from there their combinatorial meaning. This is due to the fact [21] that "with each exponential structure is associated an "exponential formula" and more generally a "convolution formula" which is an analogue of the well known exponential formula of enumerative combinatorics" [21]. Consequently with each exponential structure are associated Stirling-like, Bell-like numbers and Dobinski - like formulas are expected also, of crucial impotance for generalized coherent states' physics.

As for the another examples let us consider in more detail exponential structures.

Exponential structures. Let $\left\{Q_{n}\right\}_{n \geq 0}$ be any exponential structure and let $\{M(n)\}_{n \geq 0}$ be its denominator sequence i.e. $M(n)=$ number of minimal elements of $Q_{n}$. Let $\left|Q_{n}\right|$ be the number of elements of the poset $Q_{n}$

$$
\left|Q_{n}\right|=\sum_{\pi \in Q_{n}} 1
$$

Example: For $Q=\left\langle\Pi_{n}\right\rangle_{n \geq 1}$ where $\Pi_{n}$ is the partition lattice of $[n]$ we have $M(n)=1$. Define "Whitney-Stanley" number $S_{n, k}$ to be the number of $\pi \in Q_{n}$ of degree equal to $k \geq 1$ i.e.

$$
S_{n, k}=\sum_{\pi \in Q_{n},|\pi|=k} 1 .
$$

Define $S_{n, k}$ - generating characteristic polynomials (vide exponential polynomials) in standard way

$$
W_{n}(x)=\sum_{\pi \in Q_{n}} x^{|\pi|}=\sum_{k=1}^{n} S_{n, k} x^{k} .
$$

Then the exponential formula $\left(W_{0}(x)=1=M(0)\right)$ becomes

$$
\sum_{n=0}^{\infty} \frac{W_{n}(x) y^{n}}{M(n) n !}=\exp \left\{x q^{-1}(y)\right\},
$$

where

$$
q^{-1}(y)=\sum_{n=1}^{\infty} \frac{y^{n}}{M(n) n !} \equiv \exp _{\psi}-1
$$


with the obvious identification of $\psi$-extension choice here. Hence the polynomial sequence $\left\langle p_{n}(x)=\frac{W_{n}(x)}{M(n)}\right\rangle_{n \geq 0}$ constitutes the sequence of binomial polynomials i.e. the basic sequence of the corresponding delta operator $\hat{Q}=q(D)$. We observe then that

$$
p_{n}(x)=\sum_{k=0}^{n} \frac{S_{n, k} x^{k}}{M(n)} \equiv \sum_{k=0}^{n}\left[0,1,2, \ldots, k ; b_{n}\right] x^{k}
$$

are just exponential polynomials' sequence for the equidistant nodes case i.e. NewtonStirling numbers of the second kind $S_{n, k}^{\sim} \equiv \frac{S_{n, k}}{M(n)}$. Both numbers and the exponential sequence are being bi-univocally determined by the exponential structure $Q$. This is a special case of the one considered in [20] and we have the - what we call- NewtonStirling-Dobinski formula (notation, history and details- see [20])

$$
p_{n}(x)=\frac{1}{\exp (x)} \sum_{k=0}^{\infty} \frac{b_{n}(k) x^{k}}{k !}=\sum_{k=0}^{n}\left[0,1,2, \ldots, k ; b_{n}\right] x^{k}, \quad(N-S-D o b)
$$

where $\left\langle b_{n}\right\rangle_{n \geq 0}$ is defined by

$$
b_{n}(x)=\sum_{k=0}^{n} S_{n, k}^{\sim} x^{\underline{k}} .
$$

Note the identification $b_{n}(x)=\frac{w_{n}(x)}{M(n)}$, where

$$
w_{n}(x)=-\sum_{\pi \in Q_{n}} \mu(\hat{0}, \pi) \lambda^{|\pi|} .
$$

$\mu$ is Möbius function and $\hat{0}$ is unique minimal element adjoined to $Q_{n}$. Corresponding Bell-like numbers [20] are then given by

$$
p_{n}(1)=\frac{1}{\exp (x)} \sum_{k=0}^{\infty} \frac{b_{n}(k)}{k !}=\sum_{k=0}^{n}\left[0,1,2, \ldots, k ; b_{n}\right], \quad(N-S-\text { Bell }) .
$$

Besides those above - in Stanley's paper [21] there are implicitly present also inversedual "Whitney-Stanley" numbers $s_{n, k}$ of the first kind i.e.

$$
s_{n, k}=-\sum_{\pi \in Q_{n},|\pi|=k} \mu(\hat{0}, \pi) .
$$

On this occasion and to the end of considerations on exponential structures and Stirling like numbers let us make few remarks. q-extension of exponential formula applied to enumeration of permutations by inversions is to be find in Gessel's paper [22] (see there Theorem 5.2.) where among others he naturally arrives at the $q$-Stirling numbers of the first kind giving to them combinatorial interpretation. Recent extensions of the exponential formula in the prefab language [1] are to be find in [4]. Then note: exponential structures, prefab exponential structures (extended ones - included) i.e. schemas where exponential formula holds-imply the existence of Stirling like and Bell like numbers. As for the Dobinski-like formulas one needs binomial or extended binomial coefficients' convolution as it is the case with $\psi$-extensions of umbral calculus in its operator form.

Other Generalizations in brief. We indicate here THREE kinds of extensions of Stirling and Bell numbers - including those which appear in coherent states' applications in quantum optics on one side or in the extended rook theory on the other side. In the supplement for this brief account to follow on this topics let us note that apart from applications to extended coherent states ${ }^{6}$ physics of quantum oscillators or strings $[6-11,24,25]$ and related Feymann diagrams ${ }^{6}$ description [26] where we 
face the spectacular and inevitable emergence of extended Stirling and Bell numbers (consult also [27]) there exists a good deal of work done on discretization of space time [28] and/or Schrodinger equation using umbral methods [29] and GHW algebra representations in particular (see: $[28,29]$ for references).

\section{Acknowledgements}

Discussions with Participants of Gian-Carlo Rota Polish Seminar on all related topics http://ii.uwb.edu.pl/akk/sem/sem_rota.htm - are appreciated with pleasure.

\section{References}

[1] E. Bender, J. Goldman Enumerative uses of generating functions, Indiana Univ. Math.J. 20 1971), 753-765.

[2] D. Foata and M. Schützenberger, Th'eorie g'eometrique des polynomes euleriens, (Lecture Notes in Math., No. 138). Springer-Verlag, Berlin and New York, 1970.

[3] A. Nijenhuis and H. S. Wilf, Combinatorial Algorithms, 2nd ed., Academic Press, New York, 1978.

[4] A. K. Kwaśniewski, Cobweb posets as noncommutative prefabs Adv. Stud. Contemp. Math. vol. 14 (1), 2007 pp. 37-47.

[5] A. K. Kwaśniewski, Information on combinatorial interpretation of Fibonomial coefficients Bull. Soc. Sci. Lett. Lodz Ser. Rech. Deform. 53, Ser. Rech.Deform. 42 (2003), 39-41. ArXiv: math.CO/0402291 v1 18 Feb 2004

[6] A. K. Kwaśniewski, The logarithmic Fib-binomial formula Advanced Stud. Contemp. Math. 9 No 1 (2004), 19-26. ArXiv: math.CO/0406258 13 June 2004.

[7] A. K. Kwaśniewski, Fibonacci-triad sequences Advan. Stud. Contemp. Math. 9 (2) (2004),109-118.

[8] E. Spiegel, Ch. J. O’Donnell Incidence algebras Marcel Dekker, Inc. Basel 1997.

[9] http://mathworld.wolfram.com/HasseDiagram.html

[10] A. K. Kwaśniewski, Prefab posets ' Whitney numbers Bull. Soc. Sci. Lett. Lodz, vol 55. 2005, pp. 17-25; ArXiv:math.CO/0510027

[11] J. Konvalina, A Unified Interpretation of the Binomial Coefficients, the Stirling Numbers and the Gaussian Coefficients The American Mathematical Monthly 107 (2000), 901-910.

[12] A. K. Kwaśniewski Main theorems of extended finite operator calculus Integral Transforms and Special Functions, 14 No 6 (2003), 499-516.

[13] M. Ward: A calculus of sequences, Amer.J.Math. Vol.58, (1936), 255-266.

[14] A. K. Kwaśniewski, The logarithmic Fib-binomial formula Advanced Stud. Contemp. Math. 9 No 1 (2004), 19-26

[15] Z. Palka, A. Ruciski Lectures on Combinatorics.I. WNT Warsaw 1998 (in polish)

[16] A. K. Kwaśniewski, Fibonacci q-Gauss sequences Advanced Studies in Contemporary Mathematics 8 No 2 (2004), 121-124. ArXive: math.CO/040559131 May 2004.

[17] M. Dziemiańczuk http:www.dejaview.cad.pl/brudnopisnaukowy.php

[18] S.A. Joni ,G. C. Rota, B. Sagan From sets to functions: three elementary examples Discrete Mathematics 37 (1981), 193-2002.

[19] H.S. Wilf, Generatingfunctionology Boston: Academic Press,1990.

[20] A. K. Kwaśniewski, On umbral extensions of Stirling numbers and Dobinski-like formulas ASCM 12(2006) no. 1, pp.73-100.

[21] R. Stanley, Exponential structures, Studies in Applied Math. 59 (1978), 73-82.

[22] I.M. Gessel A q-analog of the exponential formula Discrete Math. 40 (1982), 69-80 
[23] Kent E. Morrison q-exponential families The Electronic Journal of Combinatorics 11 (2004), No R36

[24] M. A. Mendez, P. Blasiak , K. A. Penson Combinatorial approach to generalized Bell and Stirling numbers and boson normal ordering problem arXiv : quantph/0505180 May 2005

[25] A.I. Solomon , P. Blasiak, G. Duchamp , A. Horzela , K.A. Penson Combinatorial Physics, Normal Order and Model Feynman Graphs Proceedings of Symposium 'Symmetries in Science XIII', Bregenz, Austria, 2003 arXiv: quant-ph/0310174 v1 29 Oct 2003

[26] Bender, C.M, Brody, D.C, and Meister, BK Quantum field theory of partitions Journal of Mathematical Physics, 40, (1999), 3239-3245.

[27] Bender, CM, Brody, DC, and Meister, BK (2000) Combinatorics and field theory Twistor Newsletter 45, 36-39.

[28] A Dimakis, F Mller-Hoissen and T Striker Umbral calculus, discretization, and quantum mechanics on a lattice J. Phys. A: Math. Gen. 29, (1996) 6861-6876

[29] D. Levi, P. Tempesta and P. Winternitz Umbral Calculus, Difference Equations and the Discrete Schroedinger Equation J.Math.Phys. 45 (2004) 4077-4105. 